mutations of H3F3A and H3F3B. METHODS/STUDY

Clinicopathologic and Prognostic Significance of LGR5, a Cancer Stem cell Marker in Peritoneal Metastasis of a Colorectal Origin

Chukwuemeka Ihemelandu

Georgetown - Howard Universities

OBJECTIVES/SPECIFIC AIMS: Leucine-rich repeat-containing G-protein-coupled receptor 5 (LGR5) is expressed on Wnt/ $\beta$-catenindependent adult stem cell populations of the colon. Cancer stem cells are hypothesized to be the driving force behind tumor progression and metastasis, making them attractive therapeutic targets. Our aim was to analyze the clinicopathologic and prognostic significance of LGR5 expression in a cohort of colorectal cancer patients with peritoneal metastasis. METHODS/STUDY POPULATION: A total of 49 Formalin-fixed paraffin-embedded (FFPE) tissue blocks of primary or metastatic tumors and their respective normal tissues were collected from the tissue bank for time period 2009-2015. LGR5 expression was assessed at the protein level through immunohistochemical (IHC) staining of tissue microarray (TMA) constructs consisting of pairs of tumor and normal colon tissue. The correlation between LGR5 expression and clinicopathologic parameters and prognosis was assessed by statistical analysis. RESULTS/ANTICIPATED RESULTS: Of the 49 patient sample, $30(61.22 \%)$ were female vs. 19 (38.78\%) males. Age range at initial diagnosis ranged from 31.7 years to 84.4 years, with a median age of 61.29 years. Duration of follow-up ranged from $1-9$ years with a median of 5 years.LGR5 expression was higher in colorectal cancer than in normal mucosa. In univariate survival analysis overexpression of LGR5 was significantly associated with improved survival $(\mathrm{p}=0.002)$.Of significance, LGR5 positivity was an independent prognostic marker for better prognosis in a multivariate survival analysis adjusting for prognostic variables age, stage, gender, tumor histology and grade (HR 2.67. 95\% CI 1.01-7.00, P = 0.046). DISCUSSION/SIGNIFICANCE OF IMPACT: LGR5 was significantly over expressed in colorectal cancer compared to normal tissues. LGR5 was noted to be an independent prognostic variable for an improved survival outcome in colorectal cancer patients with peritoneal metastasis, making LGR5 a potential therapeutic target in colorectal cancer patients with peritoneal metastasis.

De novo germline variants in Histone 3 Family 3A (H3F3A) and Histone 3 Family $3 B$ (H3F3B) cause a severe neurodegenerative disorder and functional effects unique from their somatic mutations

Divya R Nair ${ }^{1}$ and Elizabeth Bhoj

${ }^{1}$ Children's Hospital of Philadelphia

OBJECTIVES/SPECIFIC AIMS: Histones are nuclear proteins that associate with DNA to facilitate packaging into condensed chromatin. Histones are dynamically decorated with post-translational modifications (PTMs), which regulate such processes as DNA repair, gene expression, mitosis, and meiosis. Histone 3 Family 3 (H3F3) histones (H3.3), encoded by $\mathrm{H} 3 \mathrm{~F} 3 \mathrm{~A}$ and $\mathrm{H} 3 \mathrm{~F} 3 \mathrm{~B}$, mark active genes, maintain epigenetic memory, and maintain heterochromatin and telomeric integrity. Specific somatic mutations in H3F3A and H3F3B have been strongly associated with pediatric glia and other tumors, but no germline mutations have been reported. The goal of our study was to further understand the functional effects of germline
POPULATION: We analyzed 32 patients bearing de novo germline missense mutations in $\mathrm{H} 3 \mathrm{~F} 3 \mathrm{~A}$ or $\mathrm{H} 3 \mathrm{~F} 3 \mathrm{~B}$ with core phenotypes of progressive neurologic dysfunction and congenital anomalies, but no malignancies. Patient histones were analyzed by quantitative mass spectrometry (qMS). RESULTS/ANTICIPATED RESULTS: qMS results revealed that the mutant histone proteins are present at a concentration similar to that of wild-type H3.3. qMS analysis showed strikingly aberrant PTM patterns that suggested local dysregulation. These patterns are distinct from the dominant negative somatic mutations, which cause more global PTM dysregulation. Patient cells also demonstrated upregulation of the expression of genes related to mitosis and cell division, and had a greater proliferative capacity. DISCUSSION/SIGNIFICANCE OF IMPACT: Our data suggests that the pathogenic mechanism of germline histone mutations is distinct from that of the published cancer-associated somatic histone mutations, but may converge on control of cell proliferation. Further clarification of the pathophysiology in these patients can elucidate the roles of histones and histone PTMs in human development and non-syndromic neurodegeneration. In addition, it provides a framework for targeted therapy development for this and related progressive neurologic disorders.

3177

\section{Determining mechanisms underlying hippocampal network disruption in early amyloid pathology}

Adam Caccavano ${ }^{1}$, P. Lorenzo Bozzelli, Katherine Conant and

Stefano Vicini

${ }^{1}$ Georgetown - Howard Universities

OBJECTIVES/SPECIFIC AIMS: Alzheimer's disease (AD) is the leading cause of dementia, and a rapidly growing public health crisis as life expectancy increases. Two hallmark symptoms of the disease are memory impairment and the pathological accumulation of amyloid beta protein. The hippocampus is a brain region critical for the consolidation of new memories, and one of the first regions in which amyloid accumulation is observed. Our lab and others have observed a disruption to hippocampal network activity that is critical for memory consolidation in amyloid-accumulating mice. However, the mechanisms and neuronal micro-circuitry underlying this disruption are under-explored, a critical gap that warrants exploration if we are to understand memory disruption in the disease. In this study we have investigated the hypothesis that a preferential disruption to inhibitory PV neurons and the extracellular matrix that surrounds this cell type underlies downstream network alterations. METHODS/STUDY POPULATION: We have employed the 5xFAD mouse model of familial Alzheimer's disease crossed with transgenic lines that selectively fluoresce in different neuronal sub-types. In a multi-modal approach, we have investigated behavioral, electrophysiological, and biochemical alterations between 3-monthold amyloid-accumulating 5xFAD mice and littermate controls. RESULTS/ANTICIPATED RESULTS: We observe a 35\% increase in the incidence of synchronous hippocampal oscillations known as sharp wave ripples (SWRs) in amyloid-accumulating mice versus littermate controls $(\mathrm{n}=28, \mathrm{p}=0.01)$, as well as a $95 \%$ increase in the power of slow gamma oscillations $(\mathrm{p}=0.002)$. This hyperexcitability of the hippocampal network is correlated with an impairment in hippocampal-dependent memory, assayed with the Barnes Maze, a behavioral test of spatial memory $(172 \%$ increase in latency to find escape hole, $\mathrm{n}=8, \mathrm{p}=0.01$ ). To elucidate the micro-circuitry that 